\title{
Parentalidad positiva y mindfulness: una reflexión sobre la crianza en entornos vulnerables
}

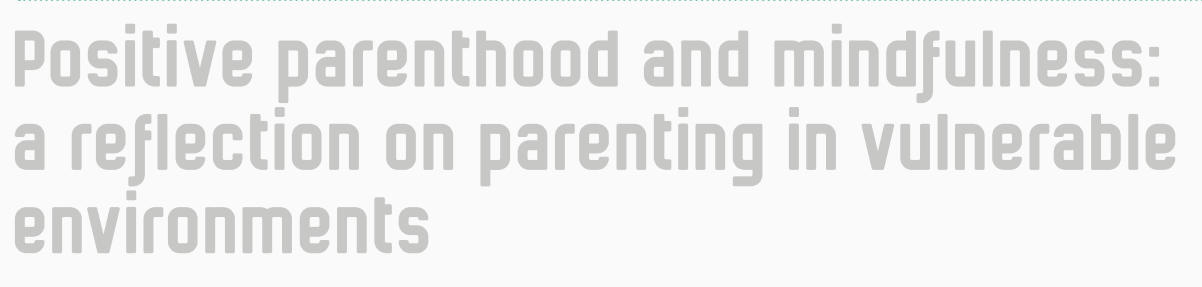

\author{
Laura Cupajita Rodriguez ${ }^{1}$ \\ Natalia Espinosa? \\ Claudia Alejandra Duque ${ }^{3}$ \\ Frank Sánchez ${ }^{4}$ \\ Edgar Muñoz ${ }^{5}$
}

(อ)

Recepción: 30/09/2020
Aprobación: 14/10/2020
(-)

Publicación: 18/12/2020

\section{Para citar este artículo:}

Cupajita Rodríguez, L., Espinosa, N., Duque, C. A., Sánchez, F., \& Muñoz, E. (2020). Parentalidad positiva y mindfulness: una reflexión sobre la crianza en entorno vulnerables. Indagare, (8), 6-21. https://doi.org/10.35707/indagare/801

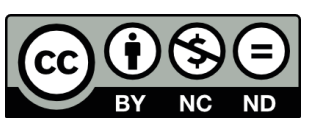

\footnotetext{
${ }^{1}$ Grupo de investigación GESE, semillero EDUCERE. Universidad de Ibagué, Colombia. ORCID: 0000-0002-0032-2398. Correo electrónico: 3220162039@estudiantesunibague.edu.co

${ }^{2}$ Grupo de investigación GESE, semillero EDUCERE. Universidad de Ibagué, Colombia. ORCID: 0000-0001-6880-9090. Correo electrónico: 3220152027@estudiantesunibague.edu.co

${ }^{3}$ Grupo de investigación GESE, semillero EDUCERE. Universidad de Ibagué, Colombia. ORCID: 0000-0002-8596-2406. Correo electrónico: alejandra.duque@unibague.edu.co

${ }^{4}$ Grupo de investigación GESE, Universidad de Ibagué, Colombia. ORCID: 0000-0003-3832-6148. Correo electrónico: frank.sanchez@unibague.edu.co

${ }^{5}$ Grupo de investigación GESE, Universidad de Ibagué, Colombia. ORCID: 0000-0002-7408-4513. Correo electrónico: edgar.m.a9602@gmail.com
} 


\section{Resumen}

La presente reflexión se derivó del proyecto de investigación titulado Validación del programa de Crianza Consciente basado en técnicas de respiración, en el cual se planteó como objetivo fomentar prácticas parentales positivas. Por lo anterior, en este artículo los autores se centraron en destacar los beneficios de la técnica de intervención con mindfulness, y su influencia sobre las habilidades parentales, especialmente en cuidadores que se encuentran expuestos a diversos factores estresores asociados a situaciones resultantes de experiencias de exclusión social. Por ello, se seleccionaron documentos del proyecto marco que motivaron la reflexión: 1) dos referentes bibliográficos que abordan la problemática relacionada con la infancia en situación de vulnerabilidad; 2) cinco estudios publicados entre 2013 y 2020 en bases de datos especializadas que exponen avances respecto al uso de la técnica mindfulness para fortalecer habilidades parentales positivas y 3 ) aportes teóricos de dos autores destacados en el campo, que dan cuenta de la relación significativa entre mindfulness como técnica de intervención y el aumento de habilidades parentales positivas aun en condiciones adversas.

\section{Abstract}

This reflection was derived from the research project entitled Validation of the conscious parenting program based on breathing techniques, in which the objective was to promote positive parenthood practices. Consequently, in this article the authors focused on highlighting the benefits of mindfulness intervention technique, and its influence on parenthood skills, especially in caregivers who are exposed to various stressors associated with situations resulting from experiences of social exclusion. For the above, documents of the framework project that motivated reflection were selected: 1) two bibliographic references that address the problem related to children in vulnerable situations; 2) five studies published between 2013 and 2020 in specialized databases that set forth advances regarding the use of mindfulness technique to strengthen positive parenthood skills and; 3) theoretical contributions from two leading authors in the field, which account for the significant relationship between mindfulness as an intervention technique and the increase of positive parenthood skills even under adverse conditions.

\footnotetext{
(A) Palabras claves: Mindfulness, parentalidad positiva, vulnerabilidad.

Key words: Mindfulness, positive parenthood, vulnerability.
} 


\section{Introducción}

De acuerdo con el Fondo de las Naciones Unidas para la Infancia (Unicef) (2001), una sociedad sostenible focaliza la atención sobre la niñez, esto implica la búsqueda de estrategias que promueven su desarrollo integral, siendo los adultos a cargo y las instituciones, actores claves para velar por su cuidado y protección. No obstante, los cuidadores (padres, madres, abuelos, etc.) pueden estar expuestos a situaciones altamente estresantes como violencia, pobreza extrema, actividades laborales demandantes, entre otras; acciones que les impide disponerse física y psicológicamente para atender de manera adecuada las diferentes necesidades de sus hijos o niños a cargo.

Con este panorama, diversos investigadores en el mundo han empezado a interesarse cada vez más en buscar soluciones para mejorar las prácticas parentales en el hogar aun cuando allí experimenten condiciones adversas. En este sentido, el mindfulness es una técnica que se empieza a implementar actualmente en los estudios científicos para apoyar el rol de la paternidad, y los resultados demuestran su eficacia para promover una crianza positiva. En la Tabla 1 se presentan los documentos y datos seleccionados del proyecto marco que fueron analizados y motivaron la reflexión en este artículo.

Tabla 1. Documentos y datos revisados

\begin{tabular}{|c|c|c|}
\hline Tipo de documento & Autores & Propósito \\
\hline \multirow[t]{5}{*}{ Institucionales } & Unicef (2014) & \multirow{5}{*}{$\begin{array}{l}\text { Analizar la problemática } \\
\text { relacionada con la infancia en } \\
\text { situación de vulnerabilidad }\end{array}$} \\
\hline & Unicef (2005) & \\
\hline & Banco Mundial \& Organización & \\
\hline & Mundial de la Salud (2015) & \\
\hline & $\begin{array}{l}\text { Código Penal Colombiano } \\
\text { (art. 229) }\end{array}$ & \\
\hline \multirow{6}{*}{$\begin{array}{l}\text { Artículos publicados en } \\
\text { revistas especializadas }\end{array}$} & Jelin (2010) & \\
\hline & Heckman (2011) & \\
\hline & Moñivas, García Diex \& García- & \\
\hline & De-Silva (2012) & \\
\hline & Acosta (2015) & \\
\hline & Beltrán (2019) & \\
\hline
\end{tabular}


INDAGARE e-ISSN: 2357-5042 • Número 8 (2020) • Universidad de Ibagué • doi: https://doi.org/10.35707/indagare/801

Aportes teóricos
sobre mindfulness y
parentalidad

Estudios publicados

entre los años 2013

y 2020 en revistas

especializadas
Kabat-Zim (2011)

Moñivas, García Diex \& García-

De-Silva (2012)

Potharst, Aktar, Rexwinkel,

Rigterink \& Bögels (2017)

García \& Grau (2017)

Parent, McKee, Rough \&

Forehand (2015)
Destacar aportes teóricos que dan cuenta de la relación significativa entre el mindfulness como técnica de intervención y el aumento de habilidades parentales positivas aún en condiciones adversas.

Fuente: elaboración propia

\section{Materiales y métodos}

Para esta revisión se seleccionaron documentos del proyecto marco que motivaron la reflexión: 1) dos referentes bibliográficos que abordan la problemática relacionada con la infancia en situación de vulnerabilidad; 2) cinco estudios publicados entre 2013 y 2020 en bases de datos especializadas que exponen avances respecto al uso de la técnica mindfulness para fortalecer habilidades parentales positivas y 3) aportes teóricos de dos autores destacados en el campo, que dan cuenta de la relación significativa entre el mindfulness como técnica de intervención y el aumento de habilidades parentales positivas aun en condiciones adversas.

\section{Resultados}

\subsection{Infancia y vulnerabilidad}

En el mundo, las actuales condiciones sociales reflejan un aumento exponencial del número de personas vulnerables que experimentan deterioro de sus condiciones. La Unicef (2014) define la vulnerabilidad como una experiencia psicosocial sujeta a la 
combinación dinámica de factores físicos y ambientales. Así, variables como la edad, el género, la pertenencia étnico-cultural, entre otras, son condiciones que al relacionarse con nuevas variables y, en general, con el contexto económico, político y cultural en el que se integran, aumentan la vulnerabilidad y el estrés parental.

La pobreza extrema es uno de los obstáculos principales que el mundo enfrenta y tiene efectos catastróficos sobre el desarrollo de la niñez. Sufrir privaciones en la primera infancia es perjudicial, se asocia con mayores problemas de salud mental y el alcance de desarrollo cognitivo superior, es decir que un niño escasamente tiene segundas oportunidades de un buen comienzo en la vida. Así lo demostró la Unicef (2005) a través de estudios realizados que confirman una relación significativa entre pobreza y desnutrición, abandono escolar, trabajo infantil y situaciones de riesgo que obstaculizan su bienestar y desarrollo en sus primeros años.

En consideración con lo anterior, la cifra de mortalidad en menores de edad ha aumentado el $5 \%$ desde el año 2000 y para 2015 fue de 5,9 millones en menores de 5 años de edad, debido a enfermedades infecciosas como neumonía, diarrea, paludismo, meningitis, tétanos, sarampión, sepsis y sida. Asimismo, se registró que las víctimas se reportan con frecuencia entre los países más desfavorecidos, por ejemplo, los índices de mortalidad más altos se encuentran en África meridional, Asia meridional y África occidental y central. Por tanto, se espera que para 2030 puedan llegar a morir 70 millones de niños antes de cumplir 5 años de edad, en particular, en países de África subsahariana, quienes tienen 12 veces más posibilidades de morir respecto a aquellos que se encuentran en contextos familiares con altos ingresos económicos. En adición, 60 millones de menores están desescolarizados, lo cual es otro efecto de la pobreza y la exclusión social (Banco Mundial \& Organización Mundial de la Salud, 2015).

De acuerdo con las cifras del Departamento Administrativo Nacional de Estadística (DANE) (2011), en Colombia para el año 2011, el 34,1 \% de los habitantes vivían en condiciones de pobreza y, entre ellos, el 10,6 \% alcanzó la pobreza extrema. Los departamentos que registraron mayor índice de pobreza fueron Chocó, Guajira, Cauca, Sucre y Córdoba. El porcentaje de pobreza de los niños y adolescentes en Colombia es de un 38,5 \% porque presentan al menos una insatisfacción moderada de sus derechos, y el 15,6 \% alcanza la pobreza extrema porque presenta privación grave de sus derechos.

En esta línea, Heckman, quien es economista y obtuvo un Premio Nobel en el año 2000, demostró a través de varios estudios la importancia de la implementación de políticas para fomentar el desarrollo humano y tuvo un enfoque claro en la infancia por ser un momento del ciclo vital que es crucial para el desarrollo. Este autor manifiesta que, en Colombia, así como en diversos países del mundo, hay discusiones fragmentadas 


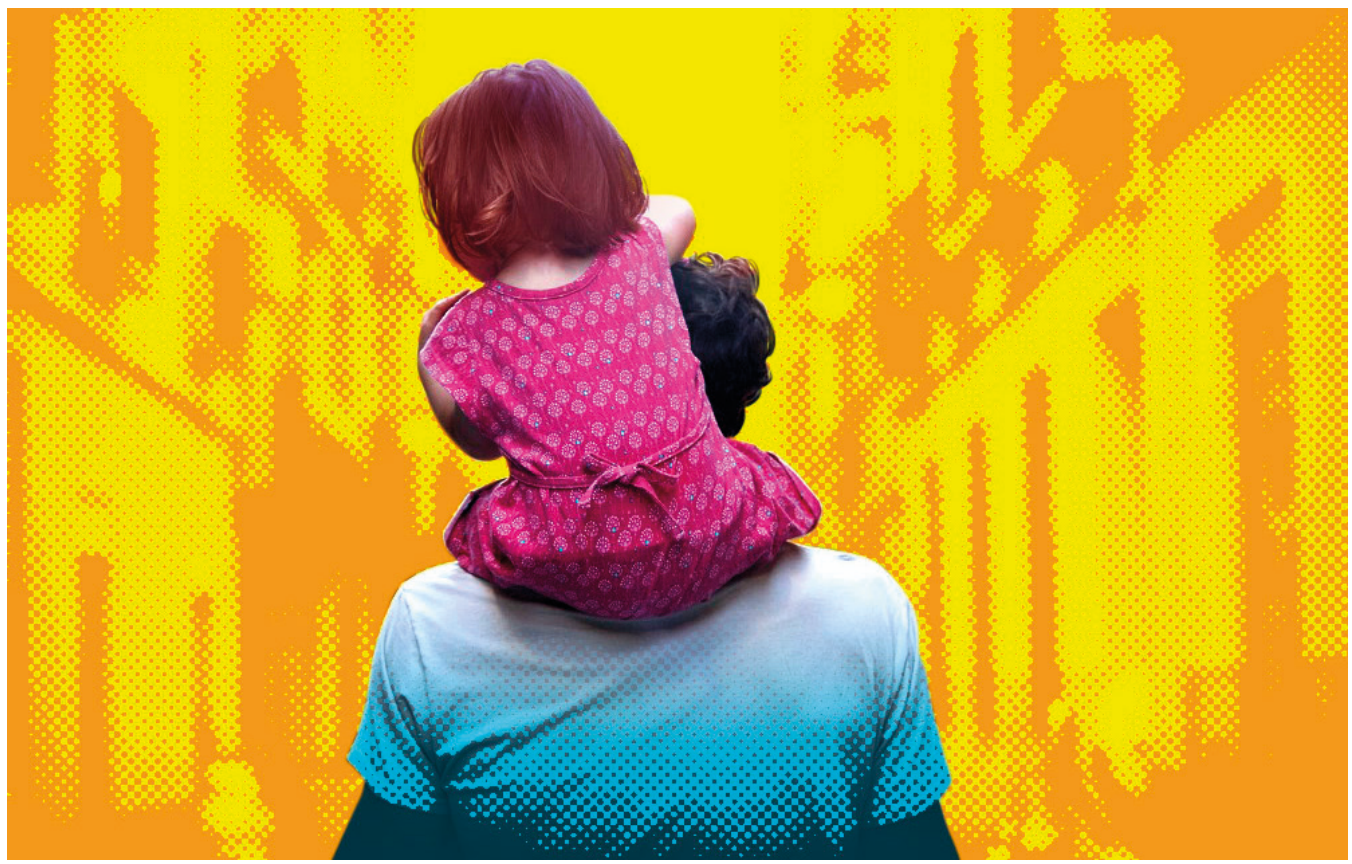

sobre política y esto se refleja en las clases sociales, así como también influye significativamente en las decisiones que se toman para el manejo de las comunidades y en cómo se estructuran las políticas públicas de salud, bienestar o enseñanza. Por otra parte, los entes gubernamentales se enfocan en solucionar las problemáticas sociales de manera progresiva y pausada; es decir, no se encaminan en todas las problemáticas en su totalidad, sino de manera selectiva, esto genera que los resultados no sean muy efectivos (Heckman, 2011).

Dicho lo anterior, Heckman (2011) enfatizó en la urgencia de poner adecuada atención sobre las diferentes políticas que actualmente se están llevando a cabo en la región colombiana, debido a que son un factor determinante para mitigar falencias en los procesos de atención que reciben los niños, en especial, aquellos que se encuentran en contextos de riesgo. En este sentido, Heckman presentó un enfoque pertinente para el abordaje de problemáticas sobre el desarrollo humano, así como la organización de estrategias que alivien la pobreza y ayuden a la próxima generación.

Por lo tanto, invertir en infancia implica el desarrollo de políticas sociales que vinculen a la familia y a los entornos educativos para garantizar el bienestar de los niños, en específico, de aquellos vinculados a entornos familiares y educativos poco estimulantes, pues dado que están en constante desarrollo, son cruciales la calidad de las 
interacciones que los adultos establecen con ellos. Se puede afirmar que las capacidades engendran habilidades, y la motivación parental les permite a los pequeños sentirse amados, aceptados y protegidos; esto aumenta la probabilidad de que pueda aprender, desarrollar sus propias destrezas y participar en la vida académica del colegio. Por el contrario, padres estresados por su alta percepción de vulnerabilidad, pueden distorsionar con más facilidad las necesidades de sus hijos o menores a cargo, así, sus respuestas no proporcionarán el bienestar que debe percibir para su pleno desarrollo (Heckman, 2011).

Se ha comprobado que niños que crecen en entornos menos favorecidos tienen menos probabilidad de éxito, por eso es que la política del siglo XXI debe tener esto presente y aprovechar todo el conocimiento para generar nuevas alternativas en los procesos de enseñanza. Por tanto, entender a la familia como centro de todo cambio es clave, puesto que los niños desarrollan a lo largo de su formación relaciones socioafectivas que tienen su origen en el núcleo familiar, de aquí la importancia de nombrar políticas de primera infancia debido a que rodean la vida del pequeño y la familia, además de reconocer los cambios que se presentan en las dinámicas familiares modernas (Heckman, 2011).

En este sentido, las políticas de desarrollo en la niñez temprana deben tener en cuenta el ambiente familiar y sus características, que puede verse amenazado por diversos factores, uno de ellos es la estructura o dinámica familiar, las necesidades afectivas y de creación de vínculos y las características de los cuidadores. Por ejemplo, se ha visto un aumento de padres o madres únicos con falta de experiencia o características propias que pueden llegar a influir negativamente sobre cómo se enseña o educa a los niños; es por esto que se sugiere evaluar cómo se están implementando las actuales políticas en Colombia, para garantizar a las familias el acompañamiento que requieren, en aras de ser garantes de los derechos de sus hijos. En otras palabras, el Estado está en la obligación de enseñar a padres, madres y otros cuidadores principales a generar un entorno de mayor calidad, como también de otorgarles herramientas que promuevan un desarrollo adecuado en los niños (Heckman, 2011).

Dicho lo anterior, la propuesta de Heckman invita a promover entornos protectores y de desarrollo para los niños y jóvenes, esto se constituye en una ruta para que la sociedad intervenga de manera oportuna y rápida en pro de fortalecer sus habilidades cognitivas y socioemocionales, en especial, de los más desfavorecidos, como estrategia para reducir la pobreza. En sus estudios, Heckman manifiesta la importancia de priorizar estas acciones y centrarse en la capacidad de determinar qué políticas son efectivas y cuáles no, situación que otorga a las familias las herramientas adecuadas para las generaciones presentes y futuras. Si esto no se realiza a tiempo, se tendrá que optar por la remediación que es más costosa en términos económicos (Heckman, 2011). 
Al ser la familia un escenario de desarrollo fundamental, es imperante hablar sobre la calidad del cuidado que allí se brinda a los niños, esto se refiere a la forma como los adultos buscan satisfacer necesidades físicas y emocionales de los pequeños. Además, estas prácticas de cuidado están permeadas por marcos normativos, económicos y sociales dentro de los cuales se desarrollan (Jelin, 2010). Por ejemplo, en contextos de pobreza urbana persisten situaciones de hostilidad y violencia, esto constituye un factor que no puede ser subestimado al buscar entender la calidad del cuidado que los adultos brindan a los niños.

\subsection{La niñez: principales víctimas de la violencia intrafamiliar}

Como se ha mencionado, la crianza en contextos de riesgo aumenta las actitudes violentas de los padres o cuidadores hacia los pequeños, esto debido a los factores asociados a la vulnerabilidad que son focos estresores para los adultos y es causa de rupturas en las relaciones al interior de la familia. Este panorama muestra que los niños terminan siendo las principales víctimas y que las experiencias adversas en los primeros años afectan su desarrollo, a saber, pueden ser causantes de que los pequeños experimenten a medida que crecen, baja autoestima, falta de empatía, depresión, estrés postraumático, problemas de conducta y aprendizaje, trastornos de sueño e irritabilidad, entre otros (Moñivas, García Diex \& García-De-Silva, 2012).

Por lo tanto, los conflictos y la violencia doméstica surgen a partir de problemas en el funcionamiento de la relación entre los cuidadores respecto a la crianza, la disciplina y los roles que cada uno asume frente al cuidado de los niños. En otras palabras, si una madre experimenta temor hacia su pareja, esto puede deteriorar su capacidad de responder de forma adecuada a las demandas de su hijo, así las necesidades básicas de los menores pueden quedar insatisfechas dado que sus rutinas de alimentación, sueño, entre otros, se alteran.

En Colombia se vive una situación de violencia intrafamiliar alarmante, puesto que se han registrado elevadas cifras de casos de violencia máxime en la última década en toda la región, que van desde la violencia de género hasta el maltrato infantil. A principios del año 2020 se registró un total de 2589 denuncias sobre casos de violencia intrafamiliar en la ciudad de Bogotá, con un aumento del $19.3 \%$ respecto al mes de enero del año 2019. También, las víctimas de violencia son en su gran mayoría las mujeres con 1958 casos (aumentó 17,5\%), en comparación con los hombres que registran 631 casos (aumentó 25,7 \%) (Rojas, 2020). 
De cara a lo expuesto, en Colombia, si la mujer es quien tiende a asumir el rol de cuidadora principal en el hogar por cuestiones culturales, ¿ cómo el Estado caracteriza situaciones de maltrato infantil vinculadas a la violencia que las mujeres experimentan? y ¿qué tan claras son las políticas colombianas sobre las acciones que se deben llevar a cabo en el entorno familiar para mitigar la violencia hacia la niñez? Si la familia supone un entorno de protección, en este país puede resultar un factor de riesgo para muchos niños, e incluso para padres o cuidadores, quienes también experimentan el estrés provocado por situaciones de vulnerabilidad que no son atendidas de forma adecuada, debido en gran parte a la exclusión social.

Como datos adicionales, en el periodo comprendido entre 2015-2019, se presentaron altos índices de violencia intrafamiliar (Acosta, 2015), con 26985 casos en Colombia, de los cuales 10435 corresponden a violencia contra niños, niñas y adolescentes con una tasa de 6747 por cada 100000 habitantes. Lo anterior refleja un panorama desalentador en función de las cifras y el aumento significativo año tras año que dan como resultado afectaciones significativas en la salud mental de las personas. Lo anterior se debe a situaciones altamente estresantes al no contar con el conocimiento para hacer uso de estrategias que les permita resolver conflictos de forma adecuada (Acosta, 2015).

En este sentido, el acto violento se concibe como el abuso de poder que ejerce un individuo sobre otro, quien es percibido como vulnerable. De hecho, la mayor parte de las víctimas de esta violencia son niños, adolescentes, mujeres, personas con capacidad reducida y adultos mayores. Resulta alarmante que la población de menor edad, que son el presente y el futuro del país sean las víctimas que a futuro deban aportar al desarrollo y la transformación social (Acosta, 2015).

Lo expuesto permite plantear cuestiones para la reflexión. Inicialmente, parece que en la actualidad la infancia constituye un problema grave en Colombia, dado que la falta de oportunidades para las familias más vulnerables les impide acceder a fuentes laborales dignas, el desplazamiento por el conflicto armado, la violencia intrafamiliar, entre otras situaciones causales del maltrato o el abandono que experimentan los niños. En suma, surgen inquietudes respecto a ¿qué tanta claridad existe sobre cómo desarrollar estas políticas para que respondan a las características de los diversos entornos familiares?, ¿qué tan efectivas están siendo estas políticas para la inclusión de la familia como entorno protector?, ¿cuál es la naturaleza de las intervenciones de los profesionales que brindan apoyo y acompañamiento a los cuidadores en el hogar para fortalecer prácticas parentales en Colombia?, ¿qué tanto conocen estas políticas las familias, los 
Por lo tanto, son diversos los retos que profesionales, investigadores y universidades que forman a los futuros profesionales deben enfrentar para que los niños no tengan que vivir experiencias adversas en sus primeros años de vida, por los efectos negativos que tendrán sobre su desarrollo emocional, neurológico, cognitivo, físico y social. El desafío es complejo y podría resumirse en una pregunta ¿qué impide el destierro de la pobreza en el país y cómo superarla? En suma, las ciencias humanas y sociales, entre las cuales se destaca a la psicología, tienen una deuda enorme con la infancia, razón por la cual las medidas para ofrecer una mirada sobre el fenómeno de la violencia hacia la niñez deben brindar la posibilidad de definir y abordar problemas específicos reconociendo al niño como un sujeto de derecho, y a la familia en sí, como un sistema prioritario de atención con necesidades básicas que no se pueden simplificar a través de medidas asistencialistas.

\subsection{Mindfulness y parentalidad positiva}

El mindfulness y la paternidad son dos aspectos ampliamente indagados y analizados en diversas investigaciones debido a la importancia que tienen las técnicas referidas a la consciencia plena en el contexto de la crianza. Los dos se encuentran estrechamente relacionados debido a la eficacia en la implementación del mindfulness sobre la crianza. En este sentido, se ha demostrado la reducción de los niveles de estrés, ansiedad, depresión y otras afectaciones referidas en los padres o cuidadores que alteran las prácticas de crianza. Además, los cuidadores que han participado en procesos de intervención a través de la técnica mindfulness reportaron una mejor capacidad de regulación emocional y conciliación con sus hijos; incluso, perciben que tienen más herramientas efectivas para el manejo de las emociones, práctica de valores, promoción de hábitos saludables y desarrollo socioafectivo de los niños.

La atención plena es de origen budista, Kabat-Zim (2011) lo describió como "la conciencia que surge al prestar atención a propósito, en el momento presente y sin prejuicios” (p. 33). En otras palabras, la atención plena es elegir y aprender a controlar la atención. Los seres humanos emiten juicios automáticos que pueden llegar a ser buenos o malos, pero también hay posibilidad de que causen distorsión de la realidad. Por tal motivo se implementa la conciencia plena porque ayuda a mejorar estas experiencias tanto internas como externas, además modera pensamientos automáticos a través de la autorregulación en el procesamiento de la información.

La Crianza Consciente (forma como se denomina en la literatura a la atención plena en la crianza) se basa en ayudar a prevenir y tratar los trastornos mentales en los niños, problemas en la crianza y la transmisión intergeneracional de los trastornos mentales 
de padres a hijos. Existen tres pilares en la intervención con mindfulness que abarcan la relación entre los pensamientos y las emociones de los cuidadores en la crianza, estos son: 1) la soberanía, 2) la aceptación y 3) la empatía. La soberanía describe la capacidad de los cuidadores para reconocer la verdadera naturaleza del hijo. La aceptación es un intento que los cuidadores hacen para comprender cómo es el niño sin querer cambiar sus potenciales y habilidades. Por último, la empatía describe la comprensión que tienen los cuidadores frente a los permanentes cambios que los niños experimentan (Moñivas, García Diex \& García-De-Silva, 2012).

Lo descrito anteriormente ha sido corroborado por investigadores que buscan brindar respuestas para fortalecer las prácticas parentales. Una investigación realizada en Estados Unidos en el año 2015 se basó en el uso de la plataforma Amazon Mechanical Turk (MTurk) en la que evaluaron tres etapas de desarrollo: infancia joven (3-7 años de edad), infancia media (8-12 años de edad) y adolescencia (13-17 años de edad). Así, la población total fue de 615 padres participantes, quienes a través de la plataforma informaron sobre procesos relacionados con su capacidad de atención plena, crianza consciente, prácticas de crianza positivas y negativas, problemas de internalización y de externalización de sus hijos (Parent, McKee, Rough \& Forehand, 2015).

Los resultados del estudio en mención permitieron afirmar que independiente de la edad de los hijos, la crianza representa un reto para los cuidadores, quienes implementan prácticas parentales positivas y negativas, que generan efectos favorables o desfavorables en el desarrollo de sus hijos. Lo anterior les permitió a los cuidadores considerar la necesidad de contar con el apoyo que proporciona la técnica de mindfulness, debido a que comprendieron que deben brindar atención al niño o adolescente sin criticar ni estresarse, guardar la calma durante las interacciones, entre otros. En los resultados manifestados por los cuidadores, luego de participar en el proceso de intervención, se destaca que sus hijos tuvieron una actitud menos resistente y las prácticas de disciplina tuvieron mayor eficacia porque hubo un aumento sustancial en los niveles de calidez y refuerzo en la interacción (Parent et al., 2015).

Por otro lado, en España se buscó reducir los niveles de ansiedad, depresión y estrés en padres que tenían a su cargo hijos con trastorno de espectro autista y parálisis cerebral. Para esto, los investigadores propusieron aumentar la capacidad de atención consciente de 11 padres (cuatro hombres y siete mujeres) con un rango de edad entre los 34 y 42 años. Como dato adicional, la mayoría de los participantes refirieron desconocer qué significa mindfulness. Los resultados encontrados fueron relevantes, debido a que se redujeron en los padres los niveles de ansiedad en un $54.94 \%$ y de estrés en un 58,01\%. Así mismo, 
aumentaron los niveles de atención consciente en un 15,54 \% razón por la cual cabe añadir que mindfulness tiene grandes beneficios en la crianza y se puede convertir en un nuevo modelo para el acercamiento a la relación paternal (Súñer, Dolz \& Alcantud, 2016).

Resulta de interés que los tratamientos basados en la técnica mindfulness para fortalecer prácticas parentales positivas muestran que, la mayoría de los cuidadores antes de participar en la intervención, manifiestan dificultades, en especial, en la manera de corregir o ejercer la disciplina, estas prácticas aumentan la interacción negativa entre padres o cuidadores con niños y adolescentes. Adicional, posterior a la intervención, los cuidadores declaran que la práctica de la crianza consciente fomenta la disminución de estrés parental y, por tanto, mejora la calidad de la interacción con sus hijos (García \& Grau, 2017).

En Colombia, se desarrolló una experiencia de investigación similar denominada Entrenamiento conductual y atención plena sobre el estrés parental y relación padre-hijo. El objetivo fue evaluar la validez mediante la relación de las variables: crianza tradicional y atención plena con el incremento de la interacción positiva. El criterio de inclusión para la selección de los participantes fue que tuvieran puntajes altos en tasas de estrés parental y puntajes bajos en la atención plena. El procedimiento consistió en entrenar a los padres sobre conductas focalizadas como observar y reforzar las acciones apropiadas de sus hijos e ignorar las inapropiadas. De esta forma les enseñaron a dar instrucciones apropiadas para que las obedecieran de manera efectiva. Los resultados fueron positivos debido a que se redujo el estrés y aumentó la calidad de la interacción entre padres e hijos (García \& Grau, 2017).

Otro estudio realizado con madres que experimentaron dificultades después del nacimiento de sus bebés examinó la efectividad de la técnica de intervención con mindfulness. Se trató de un entrenamiento grupal sobre crianza consciente de ocho semanas con 44 madres y sus bebés entre 0 y 18 meses de edad. Adicional, durante la intervención las madres se encontraban en una clínica de salud mental debido a los altos niveles de estrés, entre otros problemas. Los resultados indicaron que las madres se volvieron más conscientes en el ámbito personal y en la crianza de sus hijos, además se mostraron más compasivas consigo mismas. Se concluye que a través de la intervención con mindfulness, las madres lograron fortalecer habilidades para la regulación del estrés, estuvieron más atentas con sus bebés y con ellas mismas. Esto contribuyó a que su capacidad de respuesta fuera más efectiva para satisfacer las necesidades de sus hijos (Potharst, Aktar, Rexwinkel, Rigterink \& Bögels, 2017). 
En 2018, Moreira, Gouveia y Canavarro (2018) realizaron un estudio con 563 padres e hijos (95 madres y 61 adolescentes con una edad media de 14,26 años); a través de él encontraron que el vínculo entre la crianza consciente y el bienestar de los adolescentes está mediado por las representaciones de apego de estos últimos; es decir, la crianza consciente se asoció indirectamente con la autocompasión y la atención de los adolescentes a través de la percepción segura en la relación con los padres, y se asoció indirectamente con el bienestar de los adolescentes a través de la seguridad de apego, autocompasión y atención.

De cara a lo expuesto, el mindfulness está demostrando mediante nuevas evidencias empíricas su alto nivel de efectividad para abordar a la familia a través de la focalización de las intervenciones hacia los cuidadores, para acompañarlos de forma respetuosa en la tarea de la crianza. De nuevo llama la atención que los problemas que los cuidadores refieren en la interacción con los niños se focaliza en la disciplina. Al respecto, el mindfulness permite que, desde el afecto, la aceptación de emociones, la conexión emocional y el direccionamiento respetuoso, se asuma la disciplina como un asunto donde cuidadores, niños y adolescentes expresan emociones y conductas acordes con las diferentes edades, estas son reconocidas y tomadas en cuenta para la toma de decisiones que satisfagan a todas las partes implicadas porque se asume que todos son sujetos de derechos, incluso los niños. En suma, el mindfulness promueve la crianza desde el afecto y el respeto desde una posición contemplativa activa y disminuye el estrés que los cuidadores experimentan por diversas situaciones, hecho que ofrece alternativas prometedoras para el abordaje con familias multiestresadas por situaciones asociadas a la exclusión social.

\subsection{Crear entornos familiares protectores para la infancia en Colombia y el Tolima: ideas para la reflexión}

Dado que la niñez es un momento crucial en el ciclo vital del ser humano, se busca que su entorno ofrezca todas las herramientas necesarias para que se genere un desarrollo óptimo. Desde la perspectiva de los derechos humanos, la violencia contra la niñez en el hogar ha obligado a reflexionar cuestiones relacionadas con el bienestar humano; en este sentido, los aportes del mindfulness como estrategia de intervención focalizada hacia los cuidadores en el hogar, permite abordar la niñez como un asunto de satisfacción de necesidades básicas que no se pueden simplificar a través de procesos de atención asistencialistas que desconocen a las familias y a los niños como sujetos de derechos. Así, el compromiso del Gobierno será asegurar las bases sociales para el desarrollo de los niños y de sus entornos familiares, de modo que todos gocen de una vida digna. 
El Instituto Colombiano de Bienestar Familiar (ICBF) (2017) es una entidad del Estado encargada de velar por los derechos de los niños, entre los cuales se destaca $e l$ derecho al buen trato, esto se garantiza mediante la organización de espacios protectores que favorecen el desarrollo de la primera infancia, dado que existe una armonía en las interacciones entre las personas y el ambiente, de tal forma que los pequeños perciben en sus familias una fuente de tranquilidad y confianza. Lo anterior se ha logrado materializar de forma gradual mediante la implementación de la política pública De cero a Siempre.

Por lo tanto, es posible que si los adultos cuidadores perciben apoyo por parte de las entidades del Estado y mediante programas de acompañamiento profesional para mejorar las prácticas parentales, aumente la posibilidad de que en el hogar se fortalezcan las interacciones entre adultos y niños, debido a que los cuidadores pueden contemplarlo como un ser único, diferente a ellos, con sus propias necesidades y características, las cuales pueden o no coincidir con sus expectativas. Las evidencias descritas en este artículo invitan a los investigadores a continuar focalizando esfuerzos para apoyar a los adultos en el hogar, la escuela, entre otros escenarios educativos, por ser una fuente central para que los niños experimenten un trato respetuoso y puedan crecer con bienestar porque se sienten amados, aceptados y protegidos. De esta forma, la niñez será la base para erradicar la pobreza y construir una sociedad más justa, equitativa y sostenible.

\section{Potencial uso}

Los resultados de esta revisión permitieron ampliar la visión frente a los beneficios de la técnica de intervención con mindfulness sobre las habilidades parentales, tanto para la futura creación de programas de intervención en contextos educativos formales e informales, como para las investigaciones que se planteen profundizar y determinar la funcionalidad de estas técnicas en otros contextos.

\section{ㄴ.}

Ficha técnica del proyecto

Título del proyecto: Validación de programa de Crianza Consciente basado en técnicas de respiración.

PRIT: Educación y bienestar para el desarrollo humano integral.

Código del proyecto: 20 -010-INT.

Palabras claves: Mindfulness, infancia, educación, parentalidad positiva, vulnerabilidad.

Grupo de investigación: Educación, Salud y Sociedad (GESS). Grupo de Estudios en Educación (GESE).

Investigador principal: Eyde Frank Sánchez Quijano.

Correo electrónico: frank.sanchez@unibague.edu.co 
INDAGA3E e-ISSN: 2357-5042 • Número 8 (2020) • Universidad de Ibagué • doi: https://doi.org/10.35707/indagare/801

\section{Referencias}

Acosta, M. (2015). Comportamiento de la violencia intrafamiliar. Colombia, 2015. Recuperado de https://n9.cl/igkmz

Banco Mundial \& Organización Mundial de la Salud. (2015). Global Civil Registration and Vital Statistics: Scaling up investment Plan 2015-2024. Recuperado de https://n9.cl/by0xa

Beltrán, N. P. (2019). ¿Cuánta violencia es demasiada? Evaluación de la polivictimización en la infancia y la adolescencia. Papeles del psicólogo, 40(2), 101-108. Recuperado de http://www.papelesdelpsicologo. es/pdf/2892.pdf

DANE. (2011). Pobreza monetaria y multidimensional en Colombia 2011. Recuperado de https://n9.cl/nkpn6

García, Y. A., \& Grau, I. A. (2017). Entrenamiento conductual y atención plena sobre el estrés parental y relación padre-hijo. Psicologia: Teoria e Pesquisa, 33, 1-9. http://dx.doi.org/10.1590/0102.3772e3323

Heckman, J. (2011). El poder de los primeros años: políticas para fomentar el desarrollo humano. Infancia Imágenes, 10(1), 97-109. Recuperado de https://revistas.udistrital.edu.co/index.php/infancias/ article/view/4468

ICBF. (2017). Informe de gestión. Recuperado de https://n9.cl/xi4om

Jelin, E. (2010). Pan y afectos. La transformación de las familias. Buenos Aires, Argentina: Fondo de Cultura Económica.

Kabat-Zinn, J. (2011). Manual clínico de mindfulness. Bilbao, España: Editorial Desclée de Brouwer, S.A.

Moñivas, A., García Diex, G., \& García-De-Silva, R. (2012). Mindfulness (Atención plena): concepto y teoría. Portularia, 12, (Addenda), 83-89. Recuperado de http://hdl.handle.net/10272/5939

Moreira, H., Gouveia, M. J., \& Canavarro, M. C. (2018). Is Mindful Parenting Associated with Adolescents' Well-being in Early and Middle/Late Adolescence? The Mediating Role of Adolescents' Attachment Representations, Self-Compassion and Mindfulness. Journal of Youth and Adolescence, 47, 17711788. https://doi.org/10.1007/s10964-018-0808-7

Parent, J., McKee, L. G., Rough, J. N., \& Forehand, R. (2015). The Association of Parent Mindfulness with Parenting and Youth Psychopathology Across Three Developmental Stages. Journal of Abnormal Child Psychology, 44, 191-202. https://doi.org/10.1007/s10802-015-9978-x

Potharst, E., Aktar, E., Rexwinkel, M., Rigterink, M., \& Bögels, M. (2017). Mindful with Your Baby: Feasibility, Acceptability, and Effects of a Mindful Parenting Group Training for Mothers and Their Babies in a Mental Health Context. Mindfulness, 8, 1236-1250. https://doi.org/10.1007/s12671-0170699-9

Rojas, E. (4 de febrero de 2020). Se dispararon las cifras de violencia intrafamiliar en enero del 2020. Santa fe radio. Recuperado de https://bit.ly/3o4xFrK

Súñer, A., Dolz, L., \& Alcantud, F. (2016). Beneficios del mindfulness en la mejora del vínculo parento-filial aplicado a madres y padres de hijos con Trastorno de Espectro Autista y Parálisis Cerebral Infantil Recuperado de https://n9.cl/31kas

Unicef. (2001). Estado mundial de la infancia. Nueva York, Estados Unidos: Unicef.

Unicef. (2005). Estado Mundial de la infancia de 2006. Excluidos e invisibles. En Unicef (Ed.), Estado Mundial de la infancia de 2006. Excluidos e invisibles (pp. 156-156). New York, USA: Brodock Press.

Unicef. (2014). Vulnerabilidad y exclusión en la infancia. Madrid, España: Huygens Editorial. 